\title{
Stopping anticoagulation in a woman with unprovoked venous thromboembolism
}

\author{
Faizan Khan MSc, Grégoire Le Gal MD PhD, Marc A. Rodger MD MSc
}

— Cite as: CMAJ 2017 September 5;189:E1112-4. doi: 10.1503/cmaj.170327

A previously healthy 47-year-old woman was started on anticoagulant therapy six months ago. She had been diagnosed with a submassive pulmonary embolism after returning from a trip to the Caribbean. At that time, she had no associated deep vein thrombosis on ultrasonography and her symptoms have completely resolved without sequelae. She has not had any bleeding while on anticoagulant therapy. She asks about the need to continue anticoagulation, as she is worried about the risk of another pulmonary embolism, but is also concerned about bleeding on anticoagulant therapy. She is wondering if she could take acetylsalicylic acid (ASA) instead.

\section{What further information is needed?}

In patients with a first episode of venous thromboembolism, comprising deep vein thrombosis and pulmonary embolism, the choice of a short-term versus indefinite duration of anticoagulation is based on the index episode of venous thromboembolism. The International Society on Thrombosis and Haemostasis has recently proposed definitions that can be used in clinical practice for categorizing patients as having provoked (Box 1) or unprovoked venous thromboembolism. ${ }^{1}$ For classifying venous thromboembolism as "unprovoked," clinicians should inquire about and ensure the absence of the following provoking factors: active cancer or recent (within the previous three months) surgery, trauma or immobilization. Accordingly, this patient's pulmonary embolism was classified as unprovoked. Although many clinicians assume a flight would be a provoking factor, it is such a weak provoking factor that it is not sufficient to categorize the thrombosis as provoked. ${ }^{1,2}$

\section{What should this patient be told about the risk of recurrent venous thromboembolism if anticoagulation is stopped?}

The International Society on Thrombosis and Haemostasis suggests that it is safe to stop anticoagulants in patients in whom the risk of recurrent venous thromboembolism is less than $5 \%$ at one year following cessation of therapy. ${ }^{3}$

Nevertheless, identifying such patients is an ongoing challenge for clinicians. Some predictors of recurrent venous thromboembolism have been identified, such as gender, D-dimer levels or residual vein obstruction, but risk-stratification strategies based on individual predictors alone have failed to identify a group of low-risk patients in whom anticoagulation could safely be stopped. ${ }^{4}$

The REVERSE II, a multinational, prospective cohort study, ${ }^{5}$ validated the previously derived "Men Continue and HERDOO2" clinical decision rule (Box 2) in 2779 patients with a first unprovoked or weakly provoked major venous thromboembolism (proximal deep vein thrombosis or segmental or greater pulmonary embolism) who had completed 5-12 months of anticoagulant therapy. Of the 1213 women, 622 (51.3\%) were classified by the decision rule as having low risk of recurrence (none or one of the HERDOO points), of whom 591 stopped anticoagulant therapy. Among the 2125 patients classified as high risk by the clinical decision rule (all men, and women with two or more of the HERDOO points), anticoagulation was continued in 1802 patients, whereas 323 stopped treatment. ${ }^{5}$

The primary outcome of the 12-month incidence of symptomatic recurrent venous thromboembolism occurred in 17 of the 591 low-risk women who stopped anticoagulants (3.0\% per

Box 1: Examples of venous thromboembolism (VTE) provoked by transient or persistent risk factors*

\section{VTE provoked by transient risk factors}

Major

- Surgery with general anesthesia for more than 30 minutes

- Confined to bed (only "bathroom privileges") for at least 3 days with an acute illness

- Cesarean delivery

Minor

- Surgery with general anesthesia for less than 30 minutes

- Admission to hospital for fewer than 3 days with an acute illness

- Out of hospital, confinement to bed for at least 3 days with an acute illness

- Leg injury associated with reduced mobility for at least 3 days VTE provoked by persistent risk factors

Active cancer, defined as:

- Cancer that has not received potentially curative treatment, or

- Treatment is ongoing or

- Evidence that treatment has not been curative

*As per guidance from the International Society on Thrombosis and Haemostasis on categorization of VTE. 
Box 2: The "Men Continue and HERD002" Clinical Decision Rule

\section{All MEN with unprovoked venous thromboembolism (VTE):} continue anticoagulation

WOMEN with unprovoked VTE and 2 or more of the following features ${ }^{\star}$ present after completing 5-12 months of anticoagulation: continue anticoagulation

WOMEN with unprovoked VTE and 0 or 1 of the following features * present after completing 5-12 months of anticoagulation: discontinue anticoagulation

${ }^{\star}$ Features (in bold)

- Presence of postthrombotic signs; HER (Hyperpigmentation, Edema or Redness) in either leg.

- D-dimer level $\geq 250 \mu \mathrm{g} / \mathrm{L}$ on anticoagulants

- Obesity: body mass index $\geq 30 \mathrm{~kg} / \mathrm{m}^{2}$

- Older age: $\geq 65$ years of age

patient-year; 95\% confidence interval $[\mathrm{Cl}]$ 1.8\%-4.8\%). In highrisk patients who stopped treatment, the rate of venous thromboembolism recurrence was $8.1 \%$ per patient-year (95\% CI 5.2\%$11.9 \%)$. Conversely, the recurrence risk among high-risk patients who remained on anticoagulants was low (1.6\% per patient-year; $95 \% \mathrm{Cl} 1.1 \%-2.3 \%) .^{5}$

To date, the "Men Continue and HERDOO2" is the only prospectively validated clinical decision rule to help identify a large group of women with unprovoked venous thromboembolism, at low risk of recurrence, in whom anticoagulant therapy can safely be stopped. As such, this decision rule ${ }^{5}$ can be implemented in clinical practice.

\section{Does the patient require any investigations?}

As per the HERDOO2 rule, examination of the legs is needed to check for any signs of postthrombotic syndrome (hyperpigmentation, edema or redness in either leg). Furthermore, a D-dimer blood test should be ordered. Additionally, for patients with a first episode of venous thromboembolism who stop anticoagulation, it is important to obtain baseline imaging of the affected vascular system (e.g., compression ultrasonography of the left leg after stopping therapy in patients with initial deep vein thrombosis in the left leg). This prevents misclassification of suspected recurrent venous thromboembolism, which is important as it may lead to unnecessary lifelong anticoagulation therapy. ${ }^{7}$ Lastly, although testing for thrombophilia in the management of venous thromboembolism is controversial, patients should be tested if they are going stop anticoagulation. ${ }^{8}$ Patients with strong thrombophilias (e.g., antithrombin deficiency, antiphospholipid antibodies or combined thrombophilias, such as being homozygous for factor $\mathrm{V}$ Leiden mutation) should continue anticoagulants indefinitely.

\section{Should the patient be switched to ASA?}

In a pooled analysis of two randomized controlled trials (RCTs), ${ }^{9}$ ASA, as compared with placebo, has been shown to reduce the risk of recurrent venous thromboembolism by about $30 \%$, with- out an increase in major bleeding. However, a recent, welldesigned, double-blind RCT of 3396 patients with venous thromboembolism who had completed 6-12 months of anticoagulant therapy showed that extended anticoagulant therapy (up to 12 months) with $20 \mathrm{mg}$ rivaroxaban, as compared with ASA, was more effective at preventing recurrence (hazard ratio $0.34 ; 95 \%$ $\mathrm{Cl} 0.20-0.59, p<0.001$ ) without an increased risk of bleeding. ${ }^{10}$

\section{What should the patient watch out for?}

Patients should be reminded of signs and symptoms of recurrent venous thrombosis that should make them seek medical attention, as well as of risk factors that would warrant thromboprophylaxis. Symptoms of deep vein thrombosis include calf or thigh pain, tenderness or leg swelling, chest pain lasting more than 15 minutes, unexplained shortness of breath or palpitations as symptoms of pulmonary embolism. ${ }^{5,6}$ Patients should not delay seeking medical attention (i.e., they should be seen within hours, not days). Patients with an unprovoked venous thromboembolism in whom a new risk factor develops after they stop anticoagulation (e.g., a hospital admission) would be at high risk of recurrence during exposure to the provoking risk factor. Such patients should receive thromboprophylaxis. ${ }^{11}$

\section{Are any special precautions required for future travel?}

Although it is greater on trips longer than four hours, overall the absolute risk of travel-related venous thromboembolism is low. ${ }^{2}$ In flights longer than 12 hours, the absolute risk of severe pulmonary embolism (the most serious complication of venous thromboembolism) is about five per million. ${ }^{12}$ There are no data to support the routine use of pharmacologic thromboprophylaxis in this indication. Simple benign precautions, such as frequent ambulation, calf exercises and avoiding dehydration and sedatives are likely sufficient.

\section{Case revisited}

The HERDOO2 rule was applied: the patient's body mass index was $27 \mathrm{~kg} / \mathrm{m}^{2}$, she had no postthrombotic syndrome, and her D-dimer level was slightly elevated at $300 \mathrm{~g} / \mathrm{L}$. Because the patient had only one of the HERDOO criteria, she was classified as having a low risk of recurrence. After discussion with the patient regarding the long-term bleeding risk associated with anticoagulation, as well as the long-term risk of recurrent venous thromboembolism, a decision was made to stop anticoagulant therapy.

\section{References}

1. Kearon C, Ageno W, Cannegieter SC, et al. Categorization of patients as having provoked or unprovoked venous thromboembolism: guidance from the SSC of ISTH. J Thromb Haemost 2016;14:1480-3.

2. Watson HG, Baglin TP. Guidelines on travel-related venous thrombosis. Br J Haematol 2011;152:31-4.

3. Kearon C, lorio A, Palareti G. Risk of recurrent venous thromboembolism after stopping treatment in cohort studies: recommendation for acceptable rates and standardized reporting. J Thromb Haemost 2010;8:2313-5.

4. Rodger M, Carrier M, Gandara E, et al. Unprovoked venous thromboembolism: short term or indefinite anticoagulation? Balancing long-term risk and benefit. Blood Rev 2010;24:171-8. 
5. Rodger MA, Le Gal G, Anderson D, et al. Validating the HERDOO2 rule to guide treatment duration for women with unprovoked venous thrombosis: multinational prospective cohort management study. BMJ 2017;356:j1065.

6. Rodger MA, Kahn SR, Wells PS, et al. Identifying unprovoked thromboembolism patients at low risk for recurrence who can discontinue anticoagulant therapy. CMAJ 2008;179:417-26.

7. Hamadah A, Alwasaidi T, Le Gal G, et al. Baseline imaging after therapy for unprovoked venous thromboembolism: a randomized controlled comparison of baseline imaging for diagnosis of suspected recurrence. J Thromb Haemost 2011;9:2406-10.

8. Kearon C, Akl EA, Comerota AJ, et al. Antithrombotic therapy for VTE disease: Antithrombotic Therapy and Prevention of Thrombosis, 9th ed.: American College of
Chest Physicians Evidence-Based Clinical Practice Guidelines. Chest 2012;141: Suppl:e419S-e494S.

9. Simes J, Becattini C, Agnelli G, et al. Aspirin for the prevention of recurrent venous thromboembolism: the INSPIRE collaboration. Circulation 2014;130: 1062-71.

10. Weitz JI, Lensing A, Prins MH, et al. Rivaroxaban or aspirin for extended treatment of venous thromboembolism. N Engl J Med 2017;376:1211-1222.

11. Kearon C, Akl EA, Ornelas J, et al. Antithrombotic therapy for VTE disease: CHEST guideline and expert panel report. Chest 2016;149:315-52.

12. Kuipers S, Schreijer AJM, Cannegieter SC, et al. Travel and venous thrombosis: a systematic review. J Intern Med 2007;262:615-34.
Competing interests: Grégoire Le Gal reports acting as a co-investigator on clinical trials on behalf of Portola Pharmaceuticals, Boehringer-Ingelheim, Pfizer, GlaxoSmithKline, Bristol-Myers Squibb, LEO Pharma, Daiichi Sankyo and Bayer; he also reports receiving honoraria from Bayer, Pfizer, GlaxoSmithKline, LEO Pharma, Sanofi and bioMérieux, outside the submitted work. Marc Rodger reports receiving investigator-initiated grants from Programme hospitalier de recherche clinique and bioMérieux for a project validating the HERDOO2 clinical decision rule. No other competing interests were declared.
This article has been peer reviewed.

The clinical scenario is fictional.

Affiliations: School of Epidemiology, Public Health \& Preventive Medicine (Khan), University of Ottawa; Clinical Epidemiology Program (Khan, Le Gal, Rodger), Ottawa Hospital Research Institute; Ottawa Blood Disease Centre, Department of Medicine (Le Gal, Rodger), University of Ottawa and The Ottawa Hospital, Ottawa, Ont.
Contributors: All of the authors contributed substantially to the conception and design of the article, drafted the article, revised it critically for important intellectual content, gave final approval of the version to be published and agreed to be accountable for all aspects of the work.

Acknowledgement: All of the authors are members of the Canadian Venous Thromboembolism Clinical Trials and Outcomes Research (CanVECTOR) network.

Correspondence to: Marc Rodger, mrodger@ohri.ca 